\title{
Comparative Studies on the Diagnostic Validity of Widal and Evaluation of Interferon Gamma Response in Patients Investigated for Typhoid Fever
}

Mujahid Nura ( $\nabla$ mujahidnura81@gmail.com )

Kano University of Science and Technology Wudil https://orcid.org/0000-0003-0621-5152

Yusuf Mohammed

Bayero University Kano

Yusuf Ibrahim

Bayero University Kano

Nura Sani

Federal University Dutse

\section{Bashir Abdulkadir}

Umaru Musa Yaradua University Katsina

Mudassir Wada

Federal University Dutse

Article

Keywords: Interferon gamma, Blood culture, Widal, Typhoid, Salmonella

Posted Date: September 11th, 2020

DOl: https://doi.org/10.21203/rs.3.rs-71755/v1

License: (1) This work is licensed under a Creative Commons Attribution 4.0 International License.

Read Full License 


\section{Abstract}

Typhoid fever remains a public health challenge in developing countries. In Nigeria, patients usually come with complains of fever and other symptoms typical of febrile illnesses where resource limitation in healthcare facilities necessitates the use of screening tests for diagnosis. Widal test is widely used due to its simplicity. However, the specificity of this test has been debated. The present study compared the diagnostic validity of Widal to blood culture and evaluated interferon gamma response among the study participants. A total of 90 patients with complain of fever and other symptoms suggestive of typhoid fever were recruited for the study. Widal serology, automated blood culture and interferon gamma concentration were conducted using rapid antibody detection kit, BACTEC and sandwich enzyme linked immunosorbent assay (ELISA) respectively. Of the 90 samples tested, 63 (70.0\%) were positive for anti Typhi $O$ antigen while 42 (46.7\%) were positive for anti- Typhi H antigen. Similarly, 18 (20\%) of the patients had non-S. Typhi culture positivity while $72(80 \%)$ had no bacteria isolated. Overall, $0(0 \%)$ of the cases had S. Typhi positive culture. Fisher's exact test suggests a significant difference between blood culture status and prevalence of anti-S. Typhi $\mathrm{H}$ antigen. Interferon gamma concentration was found to be significantly associated with blood culture status as plasma interferon gamma levels increased with bacteraemia. Fisher's exact test showed a strong statistical relationship between interferon gamma concentration and blood culture $(\mathrm{p}=0.003)$. Interferon gamma has a direct proportionality with non- $\mathrm{S}$. Typhi culture as such, could be a good marker for the development of an alternative screening test, possibly an interferon gamma based detection system for typhoid fever. However, further research is required to implement that.

\section{Introduction}

Salmonella Typhi is a facultative, intracellular pathogen, which is widespread and poses a serious health challenge in developing countries (Fidan et al., 2008). World Health Organization (2018) estimates the global typhoid fever disease burden at 11-20 million cases annually, resulting in about 128,000-161,000 deaths per year. Salmonella serovars Typhi and Paratyphi are restricted to human hosts and cause systemic disease that can often be fatal (Pham and McSorley, 2015).

It is a well-known fact that typhoid fever has become endemic in developing countries including Nigeria (Chu et al., 2011). The current issue of drug resistance continues to increase at an alarming rate thus, narrowing the chemotherapeutic options available to treat this infection hence, claiming the lives of so many people with an estimated rate of 11-20 million cases annually, and resulting in about 128,000161,000 deaths per year (World Health Organization, 2018). In a recent study by Abdulkarim and Mohammed, (2017), about 1,225 cases of typhoid fever were recorded within five years in a single hospital in Kano State. Nas et al. (2017) also reported an 18\% prevalence of typhoid fever in Kumbotso local government area of Kano state. Typhoid fever causes significant morbidity and mortality in developing countries (Ajibola et al., 2018). A serious complication of typhoid fever is perforation of the intestine and remains a significant surgical problem in developing countries where patients usually perforated within 14 days of illness (Chalya et al., 2012). High incidence of perforation in most 
developing countries has been attributed to late diagnosis. The diagnosis of typhoid fever is commonly made using Widal test which has since been proven to be neither sensitive nor specific (Ajibola et al., 2018) due to cross reactivity of antibodies with similar antigens. T-cells on the other hand have single antigen specificity and hence, are not prone to cross-reactivity unlike antibodies. When primed with

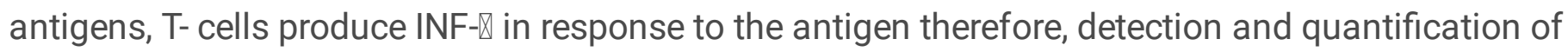


of the cytokine that may be indicative of typhoid infection. As previously documented, INF- $\$ has been used in the diagnosis of latent TB infection and found to be about 95\% specific (Siegel et al., 2018). Early and definitive diagnosis of typhoid is therefore critical in avoiding fatal complications such as perforation of the intestines (Abbas et al., 2014). This study compared the levels of Interferon gamma and anti-S. Typhi $\mathrm{O}$ and $\mathrm{H}$ with standard blood culture respectively to determine the diagnostic relevance of Widal

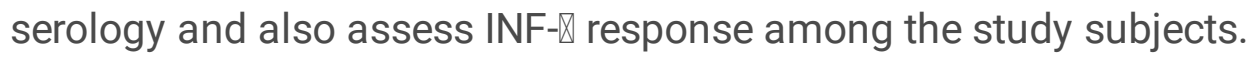

\section{Materials And Methods}

\section{Study Area}

The study was conducted at Aminu Kano teaching hospital (AKTH) and Murtala Muhammad specialist hospital (MMSH), Kano - Nigeria, where samples were collected from MMSH and analysed at AKTH.

\section{Study Population}

The study population comprised of patients with clinical signs and symptoms suggestive of typhoid fever with request of Widal test from Physicians at MMSH.

\section{Sample Size}

The sample size used in this study was determined using the formula developed by (Gberikon et al., 2019), based on previous prevalence of Salmonella Typhi infection in Kano State (4.5\%) as reported by Akinyemi et al. (2018). A final sample size of 90 was arrived at.

\section{Ethical Consideration}

Ethical approval was sought and obtained from the ethical committee, Aminu Kano teaching Hospital and Kano State Ministry of Health with reference numbers AKTH/MAC/SUB/12A/P-3/VI/2749 and MOH/Off/797/T.I/1424 respectively.

\section{Questionnaire Administration}

A structured questionnaire was administered to obtain demographic characteristics and factors associated with $S$. Typhi infection among the study population.

\section{Sample Collection and Transportation}


Using a sterile syringe, exactly $10 \mathrm{ml}$ of blood from each consented patient was aseptically collected; $7 \mathrm{ml}$ dispensed into the BACTEC aerobic plus culture vial and $3 \mathrm{ml}$ in a plain container which were both transported to the laboratory for further analysis (Andualem et al., 2014).

\section{Sample Processing}

\section{Widal Serology.}

Widal slide agglutination test was done using $S$. Typhi, S. paratyphi A, S. paratyphi B and $S$. paratyphi C O and $\mathrm{H}$ antigens according to the instructions of the manufacturer. The antigen suspension commercially available in $5 \mathrm{ml}$ volume from Chronolab systems, (Barcelona, Spain) was used. A direct qualitative slide agglutination technique was used in this study for determination of the agglutination ability of sera. (Deksissa and Gebremedhin, 2019).

\section{Inoculation of Blood Samples for Culture.}

Blood culture was done using the BACTEC fluorescent series 9120 instruments (Becton Dickinson, USA) automated microbiology systems following the method described by Anvarinejad et al. (2016) with slight modifications. Exactly $7 \mathrm{ml}$ of blood was aseptically dispensed into the BD Bactec Plus aerobic/F culture vial containing soybean-casein digest broth and then loaded into the BACTEC machine within 30 minutes of sample collection. Whenever the machine gives an alert, the specific bottle was removed, Gram stained, and sub cultured on chocolate agar and MacConkey's agar. The isolates were identified as Salmonella based on Gram staining, the oxidase test, the catalase test, motility, triple-sugar iron (TSI) fermentation, and colony morphology. Negative were removed after the system confirms them negative.

\section{Interferon Gamma Quantification}

This was done using the INF-gamma human ELISA kit from Melsin Medical Co. Limited (Cat. No. : EKHU0162), following the manufacturer's guidelines. Absorbance of each well was read on a spectrophotometer using $450 \mathrm{~nm}$ as the primary wavelength. The standard curve with standard concentration on the $x$-axis and absorbance on the $y$-axis was plotted for interferon gamma to best-fit straight line through the standard points.

\section{Data Analysis}

A Four parameter Logistic (4PL) regression was used to plot a standard curve fit from which interferon gamma concentration of samples was interpolated. Statistical association between levels of interferon gamma, anti- $S$. Typhi antibody and blood culture among the study subjects was determined at $95 \%$ confidence interval using Fisher's exact test with the aid of statistical package for social sciences (SPSS) version 22 where a $p$ value of $\leq 0.05$ was considered significant.

\section{Results}


Table 1

Prevalence of Typhoid Fever based on Widal and Blood culture.

\begin{tabular}{|llllll|}
\hline Parameters & $\begin{array}{l}\text { No. (\%) } \\
\text { Examined }\end{array}$ & $\begin{array}{l}\text { Culture Negative } \\
(\%)\end{array}$ & $\begin{array}{l}\text { Non- } \text {. Typhi Culture } \\
\text { Positive }(\%)\end{array}$ & $\begin{array}{l}\text { P- } \\
\text { Value }\end{array}$ & OR \\
\hline $\begin{array}{l}\text { Anti - Typhi } \\
\text { O }\end{array}$ & & & & & \\
\hline Positive & $63(70.0)$ & $52(57.8)$ & $11(12.2)$ & 0.358 & 1.655 \\
\hline Negative & $27(30.0)$ & $20(22.2)$ & $7(7.8)$ & & \\
\hline Total & $90(100)$ & $72(80)$ & $18(20)$ & & \\
\hline $\begin{array}{l}\text { Anti -Typhi } \\
\text { H }\end{array}$ & & & & & \\
\hline Positive & $42(46.7)$ & $38(42.2)$ & $4(4.4)$ & & \\
\hline Negative & $48(53.3)$ & $34(37.8)$ & $14(15.6)$ & & \\
\hline Total & $90(100)$ & $72(80)$ & $18(20)$ & & \\
\hline *=significant statistical difference using fisher's exact test. & & \\
\hline
\end{tabular}

Table 2

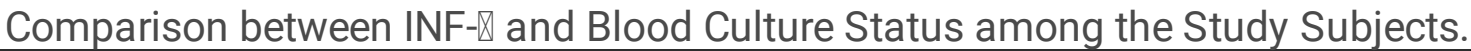

\begin{tabular}{|c|c|c|c|c|}
\hline INF-》 Conc. (pg/mL) & No. (\%) Examined & Negative (\%) & Non- S. Typhi Positive (\%) & P-Value \\
\hline$\leq 15.00$ & $21(23.3)$ & $21(23.3)$ & $0(0)$ & $0.003^{*}$ \\
\hline $15.01-20.00$ & $19(21.1)$ & $17(18.9)$ & $2(2.2)$ & \\
\hline $20.01-25.00$ & $24(26.7)$ & $18(20.0)$ & $6(6.7)$ & \\
\hline$>25.00$ & $26(28.9)$ & $16(17.8)$ & $10(11.1)$ & \\
\hline Total & $90(100)$ & $72(80)$ & $18(20)$ & \\
\hline
\end{tabular}

\section{Discussion}

Typhoid fever remains a public health problem in developing countries and its diagnosis on clinical ground is often difficult. Diagnosis in developing countries is typically done using Widal test. However, the value of the test has been of great concern.

From the present study, the prevalence of typhoid fever based on widal slide agglutination was found to be $63(70 \%)$ based on reaction to S. Typhi $O$ antigen, and 42 (46.7\%) based on reaction to S. Typhi H antigen. This is in good agreement with the result of Deksissa and Gebremedhin, (2019) where they recorded a Seroprevalence of $49.5 \%$ for both $S$. Typhi $\mathrm{O}$ and $\mathrm{H}$ antigens. However, no single $S$. Typhi 
isolate was recovered from patient's blood using blood culture which is gold standard. The findings of this research are in good agreement with that of Igiri et al. (2018) where they determined the prevalence of enteric fever in Ahmadu Bello university teaching hospital Zaria and found $70 \%$ prevalence using Widal but $0 \%$ using standard blood culture. Fisher's exact test showed a statistical difference between widal and non- $S$. Typhi blood culture $(p=0.035)$ as reported in Table 1 . This disagreement between blood culture and widal result might be due to the fact that Widal test is based on the reaction of $S$. Typhi antigens $(\mathrm{O}$ and $\mathrm{H})$ to specific antibodies in patient's serum where cross reactivity with similar antigens is possible. Cross reactivity of widal has been reported in patients with dengue fever by Bhatti et al. (2015) where they recruited patients who had clinical and serological evidence of dengue virus infection and subjected them to widal screening test. They found about 33\% of the patients with dengue fever to be widal positive with no single Salmonella Typhi bacterium isolated from their blood. A similar trend was also recorded in this research where other pathogenic bacteria were isolated from the blood of 18 (20\%) of the patients tested. High seropositivity of widal might also be due to cross reactivity of the antigens to antibodies against malaria due to its endemic nature in Nigeria, leading to misdiagnosis and treatment of the wrong disease by physicians (Igiri et al., 2018). As seen in Fig. 1, most of the patients in this study had no specific symptoms of typhoid fever like constipation, diarrhoea and stomach-ache. The results obtained in this study might give an insight to the reason for rapid increase in the emergence of drug resistant bacterial infections.

Patients are usually diagnosed and treated for enteric fever wrongly by Widal test, of which its sensitivity has been debated. Blood culture which is a gold standard in typhoid fever diagnosis is not always available and, when available, it takes about 3 to 5 days. As a result, diagnosis may be delayed or overlooked and patients without enteric fever may receive unnecessary and inappropriate antimicrobial treatment due to the heavy dependence of rapid diagnosis using clinical features and serological methods (Deksissa and Gebremedhin, 2019). Therefore, rapid tests with better sensitivity and specificity are needed for the diagnosis of enteric fever.

This study found a strong correlation between plasma interferon gamma levels and bacteraemia among the study participants. A total 21 (23.3\%) patients with interferon gamma concentration of $\leq 15 \mathrm{pg} / \mathrm{mL}$ had no bacteria isolated from blood while prevalence of bacteraemia increased as interferon gamma concentration increases with patients having INF- $y$ concentration $>25 \mathrm{pg} / \mathrm{mL}$ recording the highest prevalence of $11.1 \%$ (Table 2). This shows that blood culture status is directly proportional to interferon gamma concentration.

\section{Conclusion}

Widal serology is widely used in the laboratory diagnosis of typhoid fever which is often misleading due to cross reactivity and other factors as seen in this research. The prevalence of typhoid using Widal test was relatively high but blood culture confirmed that none of the study participants truly have active salmonella infection where on the other hand, interferon gamma has a direct proportionality with blood culture status as such, could be a good marker for the development of an alternative screening test, 
possibly an interferon gamma based detection system for typhoid fever. However, further research is required to implement that.

\section{Declarations}

\section{ACKNOWLEDGEMENTS}

The authors wish to acknowledge the support rendered by the management and staff of AKTH and MMSH as well as the patients investigated for typhoid fever who willingly participated in this study.

\section{References}

Abbas, H. H., Ali, K. H., Ibrahem, D. M., Ammar, F., Abd-alwahed, M. T., and Hafedh, H. (2014). Comparison study between typhoid fever and tuberculosis patients to induce and produce of Th-1 cytokines ( IL-18 and IFN- $\mathrm{Y}$ ). Europian Journal of Experimental Biology, 4(3):20-26.

Abdulkarim, I. A., and Mohammed, M. U. (2017). Spatio-temporal trends of typhoid fever among youths attending Muhammad Abdullahi Wase Specialist Hospital in Kano Metropolis, Nigeria. Bayero Journal of Pure and Applied Sciences, 10(2): 115.

Ajibola, O., Mshelia, M. B., Gulumbe, B. H. and Eze, A. A. (2018). Typhoid Fever Diagnosis in Endemic Countries: A Clog in the Wheel of Progress? Medicina (Kaunas, Lithuania), 54(2): 1-12.

Akinyemi, K. O., Oyefolu, A. O. B., Mutiu, W. B., Iwalokun, B. A., Ayeni, E. S., Ajose, S. O., and Obaro, S. K. (2018). Typhoid fever: Tracking the trend in Nigeria. American Journal of Tropical Medicine and Hygine, 99(3): 41-47.

Andualem, G., Abebe, T., Kebede, N., Gebre-Selassie, S., Mihret A. and Alemayehu, H. (2014). A comparative study of Widal test with blood culture in the diagnosis of typhoid fever in febrile patients. BMC Research Notes, 7(653): 1-6.

Anvarinejad, M., Pouladfar, G. R., Pourabbas, B., Amin Shahidi, M., Rafaatpour, N., Dehyadegari, M. A. (2016). Detection of Salmonella spp. with the BACTEC 9240 Automated Blood Culture System in 2008 2014 in Southern Iran (Shiraz): Biogrouping, MIC, and Antimicrobial Susceptibility Profiles of Isolates. Jundishapur Journal of Microbiology, 9(4): 1-6.

Bhatti, A. B., Ali, F., and Satti, S. A. (2015). Cross-Reactivity of Rapid Salmonella Typhi IgM Immunoassay in Dengue Fever Without Co-Existing Infection. Cureus, 7(12):1-9.

Chalya, P. L., Mabula, J. B., Koy, M., Kataraihya, J. B., Jaka, H., Mshana, S. E. (2012). Typhoid intestinal perforations at a University teaching hospital in Northwestern Tanzania: A surgical experience of 104 cases in a resource-limited setting. World Journal of Emergency Surgery, 7(1): 4. 
Deksissa, T., and Gebremedhin, E. Z. (2019). A cross-sectional study of enteric fever among febrile patients at Ambo hospital: prevalence, risk factors, comparison of Widal test and stool culture and antimicrobials susceptibility pattern of isolates. BMC Infectious Diseases, 19(288): 1-12.

Fidan, I., Yesilyurt, E., Gurelik, F. C., Erdal, B., and Imir, T. (2008). Effects of recombinant interferon-y on cytokine secretion from monocyte-derived macrophages infected with Salmonella typhi. Comparative Immunology, Microbiology and Infectious Diseases, 31(6): 467-475.

Gberikon, G. M., Olabode, V. B., and Ogbonna, I. O. (2019). Determination of Antibiotic Resistance of Salmonella and Molecular Confirmation of Drug Resistance Salmonella Species Isolated From Raw Chicken and Quail Eggs from Selected Farms in Jos, International Journal of Innovative Research and Development, 8(1): 37-42.

Igiri, B. E., P.C., I., G.C., E., Jimoh, O., M.A., S., and G.O, I. (2018). Diagnostic investigations and prevalence of enteric fever in Ahmadu Bello University Teaching Hospital Shika-Zaria ,. Clinical Microbiology and Infectious Diseases, 3(1): 1-5.

Nas, F. ., Ali, M., and Yahaya, A. (2017). Malaria and Typhoid Fever Co-Infection Co Infection Among Febrile Patients in. Bayero Journal of Pure and Applied Sciences, 10(2): 122-125.

Pham, O. H., and McSorley, S. J. (2015). Protective host immune responses to Salmonella infection. Future Microbiology, 10(1): 101-110.

World Health Organization. (2018). WHO | Typhoid. Retrieved March 23, 2019, from https://www.who.int/mediacentre/factsheets/typhoid/en/

\section{Figures}

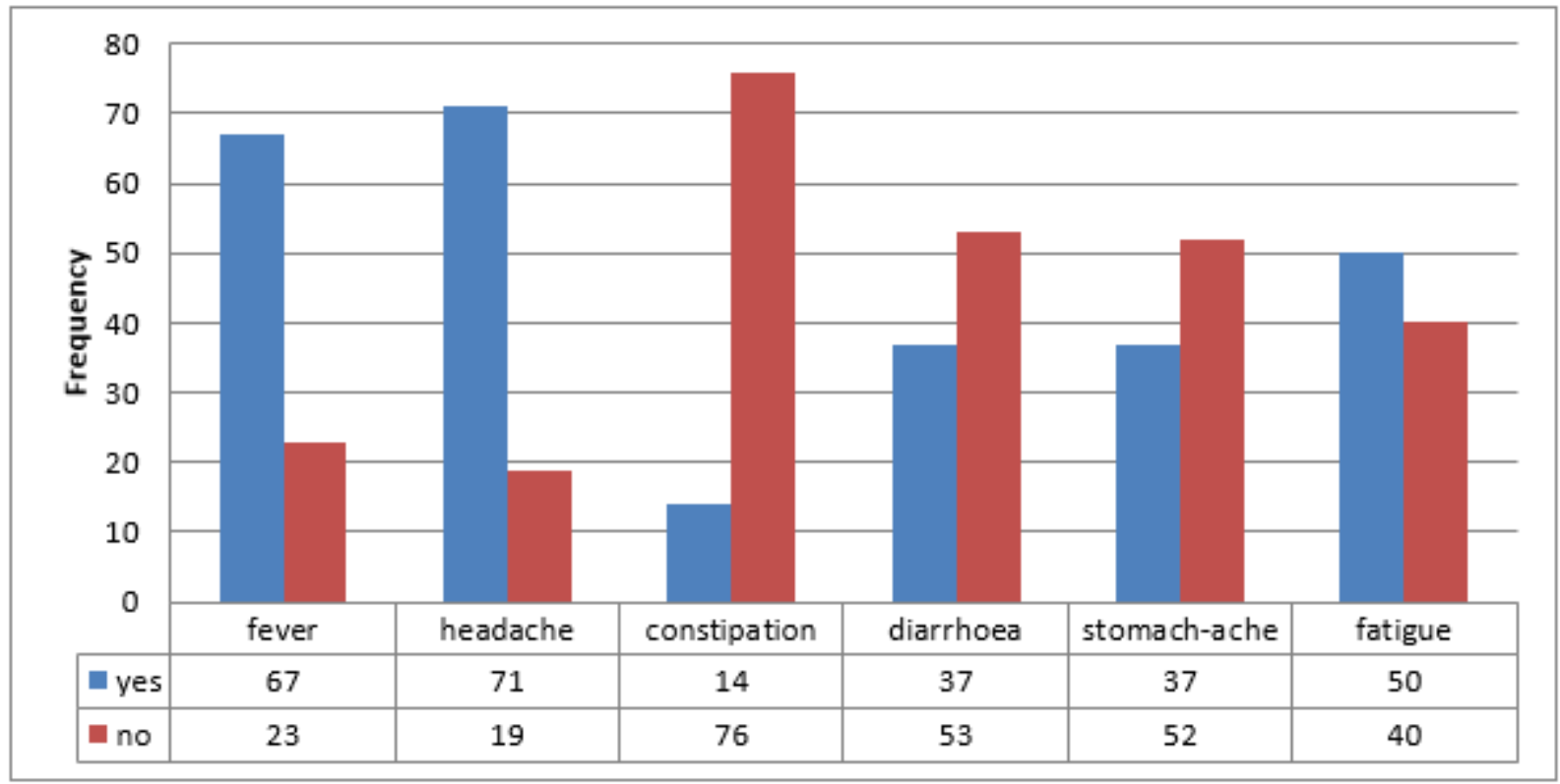


Figure 1

Clinical Presentation among patients Investigated for Typhoid Fever. 